\title{
A UNIFIED TREATMENT OF SOME THEOREMS ON POSITIVE MATRICES
}

\author{
GÉRARD LETAC
}

\begin{abstract}
Various theorems on positive matrices are shown to be corollaries of one general theorem, the proof of which bears on Legendre functions, as used in Rockafellar's Convex analysis.
\end{abstract}

1. Introduction: The main theorem. Let $X$ be a finite set, $(\mu(x))_{x \in X}$ strictly positive numbers, and $H$ a fixed linear subspace of $\boldsymbol{R}^{X}$. We shall prove the following:

THEOREM 1. There exists a unique (nonlinear) map from $\boldsymbol{R}^{X}$ to $H$, denoted $f \mapsto h_{f}$, such that

for all $g$ in $H$.

$$
\sum_{x \in X}\left[\exp f(x)-\exp h_{f}(x)\right] g(x) \mu(x)=0
$$

2. A first application: Matrices with prescribed marginals. Given an $n$-sequence $r=\left(r_{i}\right)_{i=1}^{n}$ and an $m$-sequence $s=\left(s_{j}\right)_{j=1}^{m}$ of nonnegative numbers such that $\sum_{i=1}^{n} r_{i}=\sum_{j=1}^{m} s_{j}$, denote by $\mathscr{M}(r, s)$ the set of $(n, m)$ matrices $\left(a_{i j}\right)$ with $a_{i j} \geqq 0$ such that $r_{i}=\sum_{j=1}^{m} a_{i j}$ and $s_{j}=\sum_{i=1}^{n} a_{i j}$ for all $i=1,2, \cdots, n$ and $j=1,2, \cdots, m$. Also, let $E=\{1,2, \cdots, n\}$ and $F=\{1,2, \cdots, m\}$. We define the linear map $c$ from $\boldsymbol{R}^{E} \oplus \boldsymbol{R}^{F}$ to $\boldsymbol{R}^{E \times F}$ by:

$$
c\left[\left(b_{i}\right)_{i \in E},\left(b_{j}^{\prime}\right)_{j \in F}\right]=\left(b_{i}+b_{j}^{\prime}\right)_{(i, j) \in E \times F^{\prime}} .
$$

If $X$ is a subset of $E \times F, \pi$ denotes the canonical map from $R^{E \times F}$ to $R^{X}$, i.e.

$$
\pi\left[\left(a_{i j}\right)_{(i, j) \in E \times F}\right]=\left(a_{i j}\right)_{(i, j) \in X} .
$$

We say also that $X$ is an $(r, s)$ pattern if there exists $\left(a_{i j}\right)$ in $\mathscr{M}(r, s)$ such that $X=\left\{(i, j) ; a_{i j}>0\right\}$.

Now the first corollary to the Theorem 1 is:

COROLlARY 1. Let two sequences $r=\left(r_{i}\right)_{i=1}^{n}$ and $s=\left(s_{j}\right)_{j=1}^{m}$ of nonnegative numbers with $\sum_{i=1}^{n} r_{i}=\sum_{j=1}^{m} s_{j}, M$ an $(n, m)$ matrix with $\mu_{i j} \geqq 0$ and $X=\left\{(i, j) ; \mu_{i j}>0\right\}$.

Received by the editors February 6, 1973.

AMS (MOS) subject classifications (1970). Primary 15A48; Secondary 58C20, 94A15.

Key words and phrases. Positive matrices, entropy, gradient.

(c) American Mathematical Society 1974 
Then there exist two diagonal matrices

and

$$
D_{b}=\left(e^{b_{1}}, e^{b_{2}}, \cdots, e^{b_{n}}\right)
$$

$$
D_{b^{\prime}}^{\prime}=\left(e^{b_{1}^{\prime}}, \cdots, e^{b_{m}^{\prime}}\right)
$$

with positive diagonal elements such that $D_{b} M D_{b}^{\prime}$, is in $\mathscr{M}(r, s)$ if and only if $X$ is an $(r, s)$ pattern. Furthermore, the set $\left(b, b^{\prime}\right)$ of $\boldsymbol{R}^{E} \oplus \boldsymbol{R}^{F}$, such that $D_{b} M D_{b}^{\prime}$ is in $\mathscr{M}(r, s)$, is exactly a translate of $\operatorname{Ker}[\pi \circ c]$ if nonempty.

Proof. If $b$ and $b^{\prime}$ exist, the fact that $X$ is an $(r, s)$ pattern is obvious. Conversely, suppose that $X$ is an $(r, s)$ pattern. Then there exists $\left(a_{i j}\right) \in$ $\mathscr{M}(r, s)$ with $X=\left\{(i, j) ; a_{i j}>0\right\}$. Denote $f_{i j}=\log \left(a_{i j} / \mu_{i j}\right)$ when $(i, j) \in X$ and apply the theorem to this $f \in \boldsymbol{R}^{X}$ and to $H$, the range in $\boldsymbol{R}^{X}$ of $\pi \circ c$. Then there exists $h=\left(h_{i j}\right)_{(i, j) \in X}$ in $H$ such that

$$
\sum_{(i, j) \in X} a_{i j} g_{i j}=\sum_{(i, j) \in X} \exp \left(h_{i j}\right) g_{i j} \mu_{i j}
$$

for all $\left(g_{i j}\right)_{(i, j) \in X}$ of $H$, and such $h$ is unique. Writing now $h_{i j}=b_{i}+b_{j}^{\prime}$ for some $\left(b_{i}\right)_{i=1}^{n} \in R^{E}$ and $\left(b_{j}^{\prime}\right)_{j=1}^{m} \in R^{F}$, we have $\left(e^{b_{i}} \mu_{i j} e^{b^{\prime}{ }_{j}}\right)_{(i, j) \in E} \in \mathscr{H}(r, s)$.

All suitable $\left(b, b^{\prime}\right)$ must satisfy $\pi \circ c\left(b, b^{\prime}\right)=h$, and this ends the proof.

In order to complete Corollary 1 we have to specify $\operatorname{Ker}[\pi \circ c]$ for a given $X \subset E \times F$. We index $E$ and $F$ such that $E \cap F=\varnothing$. We consider the linear graph (nonoriented) with $E \cup F$ as the set of vertices and $X$ as the set of edges. The connected components of that linear graph can be written $\left(E_{\alpha} \cup F_{\alpha}\right)_{\alpha \in A}$, where $A$ is some index set (with $E_{\alpha} \cup F_{\alpha}$ nonempty, but $E_{\alpha}$ or $F_{\alpha}=\varnothing$ can happen), and we call $\left(E_{\alpha}\right)_{\alpha \in A}$ and $\left(F_{\alpha}\right)_{\alpha \in A}$ the partitions of $E$ and $F$ associated to $X$.

Proposition 1. Let $X$ be a subset of $E \times F, \pi$ the canonical map from $\boldsymbol{R}^{E \times F}$ to $\boldsymbol{R}^{X},\left(E_{\alpha}\right)_{\alpha \in A}$ and $\left(F_{\alpha}\right)_{\alpha \in A}$ the associated partitions of $E$ and $F$. Then $\operatorname{dim}[\operatorname{Ker}(\pi \circ c)]=$ number of $\alpha$ such that $E_{\alpha} \times F_{\alpha}$ is not empty. Furthermore, when $\left(i_{1}, j_{1}\right)$ and $\left(i_{2}, j_{2}\right)$ are in $E_{\alpha} \times F_{\alpha}$ and $\left(b, b^{\prime}\right) \in \operatorname{Ker}[\pi \circ c]$, then

$$
b_{i_{1}}=b_{i_{2}}=-b_{j_{1}}=-b_{j_{2}} \text {. }
$$

Proof. Suppose $E_{\alpha} \times F_{\alpha}$ is nonempty; take $\left(i_{1}, j_{1}\right)$ and $\left(i_{2}, j_{2}\right)$ in $E_{\alpha} \times F_{\alpha}$ and $\left(b, b^{\prime}\right)$ in $\operatorname{Ker}(\pi \circ c)$. From the definition of $E_{\alpha} \cup F_{\alpha}$, either there exists a sequence of $X$

$$
\left(e_{0}, f_{0}\right),\left(e_{1}, f_{0}\right),\left(e_{1}, f_{1}\right),\left(e_{2}, f_{1}\right), \cdots,\left(e_{n}, f_{n-1}\right)
$$

or there exists a sequence of $X$

$$
\left(e_{0}, f_{0}\right),\left(e_{0}, f_{1}\right),\left(e_{1}, f_{1}\right),\left(e_{1}, f_{2}\right), \cdots,\left(e_{n}, f_{n}\right)
$$

with $e_{0}=c_{1}$ and $e_{n}=c_{2}$. 
Since $b_{e}+b_{f}^{\prime}=0$ when $(e, f) \in X$ this implies that $b_{i_{1}}=b_{i_{2}}$. In the same way we prove $b_{j_{1}}^{\prime}=b_{j_{2}}^{\prime}$. To see that $b_{i_{1}}=-b_{j_{1}}^{\prime}$, we find a path from $i_{1}$ to $j_{1}$ in an analogous manner. Then $\operatorname{dim} \operatorname{Ker}[\pi \circ c]$ is not larger than the number of nonempty $E_{\alpha} \times F_{\alpha}$. From this, it is easy to finish the proof.

Remarks. Corollary 1 was conjectured in 1964 by P. Thionet who is a French statistician [10], and in a weaker form in 1960 [9]. Proofs in particular cases are given from [1]. Independently, the case $n=m, r_{i}=s_{j}=1$ was intensively studied in a paper by R. Sinkhorn in 1964 [7]; see [3] for a recent proof and references. ${ }^{1}$

It is easy to prove the following: $X$ is an $(r, s)$ pattern if and only if for any $x \in X$ there exists an $(r, s)$ pattern $Y(x)$ such that $x \in Y(x) \subset X$. An interesting feature of the case of doubly stochastic matrices is that the patterns $Y(x)$ are associated in a natural way to permutation matrices, permitting a characterisation, via a König theorem, of a corresponding pattern as a direct sum of so-called fully indecomposable matrices [3]. It would be interesting to get such a combinatorial characterisation for a general $(r, s)$ pattern.

3. Second application: A theorem due to D. J. Hartfiel. Let $E=\{1, \cdots, n\}$ and $\mathscr{M}_{s}$ be the set of $n$-square matrices $\left(a_{i j}\right)$ with $a_{i j} \geqq 0$ and $\sum_{j=1}^{n} a_{i j}=\sum_{j=1}^{n} a_{j i}$ for all $i$ in $E$. We define the linear map $d$ from $\boldsymbol{R}^{E}$ to $R^{E \times E}$ by:

$$
d\left[\left(b_{i}\right)_{i \in E}\right]=\left(b_{i}-b_{j}\right)_{(i, j) \in E \times E} .
$$

If $X$ is a subset of $E \times E, \pi$ is the canonical map from $\boldsymbol{R}^{E \times E}$ to $\boldsymbol{R}^{X}$. We say also that $X$ is a symmetric pattern if there exists $\left(a_{i j}\right)$ in $\mathscr{M}_{s}$ such that $X=\left\{(i, j) ; a_{i j}>0\right\}$. The second corollary of Theorem 1 is essentially in D. J. Hartfiel [2].

Corollary 2. Let $M=\left(\mu_{i j}\right)$ be an $n$-square matrix with $\mu_{i j} \geqq 0$ and $X=\left\{(i, j) ; \mu_{i j}>0\right\}$. Then there exists one diagonal matrix $D_{b}=\left(e^{b_{1}}, e^{b_{2}}, \cdots\right.$, $\left.e^{b_{n}}\right)$ with positive diagonal elements such that $D_{b} M D_{b}^{-1}$ is in $\mathscr{M}_{s}$, if and only if $X$ is a symmetric pattern. Furthermore, the set of $b \in \boldsymbol{R}^{E}$ such that $D_{b} M D_{b}^{-1}$ is in $\mathscr{M}_{s}$ is exactly a translate of $\operatorname{Ker}[d \circ \pi]$ if nonempty.

Proof. Easy, taking $H=\pi \circ d\left[\boldsymbol{R}^{E}\right]$.

If we want now to complete the Corollary 2, we have to characterise $\operatorname{Ker}[\pi \circ c]$ for a given $X \subset E \times E$ and characterise symmetric patterns.

${ }^{1}$ Note ADDED IN PROOF. Professor Caussinus from the University of Toulouse has indicated to the author that a complete story of Corollary 1 can be found in Chapter 4 of M. Bacharach's monograph, Biproportional matrices and input-output change, Cambridge University Press, 1970. The first proof of Corollary 1 must be credited to W. M. Gorman (1963). 
We consider the oriented graph with $E$ as the set of vertices and $X$ as the set of arcs.

Proposition 2. $X$ is a symmetric pattern if and only if for any $e \in E$ there exists a sequence $e_{0}, e_{1}, \cdots, e_{n}$ in $E$ such that $e_{0}=e_{n}=e$ and $\left(e_{i-1}\right.$, $\left.e_{i}\right) \in X$ for $i=1, \cdots, n-1 \quad(n \geqq 1)$ (in terms of the graph $(E, X)$ every vertex belongs to a circuit).

Now we suppose that $X$ is a symmetric pattern and we partition the set of vertices of the graph $(E, X)$ into connected components $\left(E_{\alpha}\right)_{\alpha \in A}$ where $A$ is some index set and $E_{\alpha} \neq \varnothing$. These components correspond to "irreducible matrices" of [2]. We say that $E_{\alpha}$ is the partition of $E$ associated to the symmetric pattern $X$.

Proposition 3. Let $X$ be a symmetric pattern of $E \times E,\left(E_{\alpha}\right)_{\alpha \in A}$ the associated partition of $E$. Then $\operatorname{dim}[\operatorname{Ker}(\pi \circ d)]=$ number of elements of $A$. Furthermore $b \in \operatorname{Ker}(\pi \circ d)$ and $i_{1}$ and $i_{2} \in E_{\alpha}$ implies $b_{i_{1}}=b_{i_{2}}$.

Proof. Similar to Proposition 1.

4. Other applications. Following the same vein, we may now consider the result of $\mathrm{R}$. Sinkhorn [8], stating that if $M$ is an $(n, n)$ symmetric matrix with strictly positive coefficients there exists one diagonal matrix $D$ with positive diagonal coefficients such that $D M D \in \mathscr{M}(r, r)$, where $r=\left(r_{i}\right)_{i=1}^{n}$ is given (Marcus and Newman [4] consider the doubly stochastic case).

Denote $E=\{1, \cdots, n\}$ and define the linear map $e$ from $R^{E}$ to $R^{E \times E}$ by:

$$
e\left[\left(b_{i}\right)_{i \in E}\right]=\left(b_{i}+b_{j}\right)_{(i, j) \in E \times E} .
$$

If $X$ is a subset of $E \times E$, call $X$ an r-pattern if there exists a symmetric matrix $\left(a_{i j}\right)$ of $\mathscr{M}(r, r)$ such that $X=\left\{(i, j) ; a_{i j}>0\right\}$.

COROllary 3. Let $M=\left(\mu_{i j}\right)$ be a symmetric matrix with $\mu_{i j} \geqq 0$, $r=\left(r_{i}\right)_{i=1}^{n}$ a sequence such that $r_{i} \geqq 0$ and $X=\left\{(i, j) ; \mu_{i j}>0\right\}$. Then there exists one diagonal matrix $D_{b}=\left(e^{b_{1}}, \cdots, e^{b_{n}}\right)$ with positive diagonal elements such that $D_{b} M D_{b}$ is in $\mathscr{M}(r, r)$ if and only if $X$ is an r-pattern. Furthermore, the set of $b \in R^{E}$ such that $D_{b} M D_{b}$ is in $\mathscr{M}(r, r)$ is exactly a translate of $\operatorname{Ker}[e \circ \pi]$ if nonempty.

Proof. Easy, taking $H=r \circ e\left[\boldsymbol{R}^{E}\right]$.

A similar study of dimension of $\operatorname{Ker}[\pi \circ e]$ can be done considering the number of components of the graph $(E, X)$; characterisation of $r$-patterns depends on $r$ in an unknown way, except in the doubly stochastic case, which is easy to work. 
A trivial application of Theorem 1 is conditional expectation on a finite probability space: Let $\left(X_{\alpha}\right)_{\alpha \in A}$ be a partition of $X$; consider the boolean algebra $\mathfrak{A}$ generated by $\left(X_{\alpha}\right)_{\alpha \in A}$ and take $H$ as space of $\mathfrak{A}$-measurable functions.

5. Proof of Theorem 1. Consider the function $F$ defined on $H$ by:

$$
F(h)=\sum_{x \in X} \mu(x) \exp h(x) .
$$

Since $\mu(x)>0$ for all $x, F$ is strictly convex. One can easily check that $F$ is continuously differentiable and that grad $F_{h}$, element of the dual space $H^{*}$ of $H$, is given by:

$$
\operatorname{grad} F_{h}(g)=\sum_{x \in X} \mu(x) g(x) \exp h(x)
$$

for all $g$ in $H$. Now to each $f$ of $\boldsymbol{R}^{X}$, we associate one point $h_{f}^{*}$ of $H^{*}$ defined by:

$$
h_{f}^{*}(g)=\sum_{x \in X} \mu(x) g(x) \exp f(x) \text { for all } g \in H .
$$

We have to show that there exists one and only one point $h_{f} \in H$ such that $h_{f}^{*}=\operatorname{grad} F_{h_{f}}$.

Now we introduce the conjugate function $F^{*}$ of $F$, valued in $R \cup\{+\infty\}$ and defined on $H^{*}$ by:

$$
F^{*}\left(h^{*}\right)=\sup _{h \in H}\left[h^{*}(h)-F(h)\right]
$$

We denote by $C^{*}$ the interior of $\left\{h^{*} ; F^{*}\left(h^{*}\right)<\infty\right\}$. We use the following inequality:

$$
b e^{a}-e^{b} \leqq a\left(e^{a}-1\right) \quad(a, b \in \boldsymbol{R})
$$

(to see this, replace $b$ with $a+b$ ) to get $F^{*}\left(h_{f}^{*}\right) \leqq \sum_{x \in X} \mu(x) f(x)\left(e^{f(x)}-1\right)$ for all $f$ in $\boldsymbol{R}^{X}$. Clearly the range of $f \mapsto h_{f}^{*}$ in $H^{*}$ is an open set, so $h_{f}^{*} \in C^{*}$. We made an appeal to Rockafellar [6, p. 258, Theorem 26.5]: we have shown that $(H, F)$ and $\left(C^{*}, F^{*}\right)$ are convex functions of Legendre type; from the quoted theorem we can assert that the map $h \mapsto \operatorname{grad} F_{h}$ from $H$ to $H^{*}$ is injective and that the range of this map is $C^{*}$; this ends the proof.

6. Other results and remarks. The trivial example of conditional expectations quoted at the end of $\S 4$ leads us to ask if $P_{H}: R^{X} \rightarrow H$ defined by $P_{H} f=h_{f}$ by means of Theorem 1 has some properties of ordinary linear projection.

Theorem 2. If $P_{H}: R^{X} \rightarrow H$ is defined by $P_{H} f=h_{f}$ and if $H$ and $H^{\prime}$ are two linear subspaces of $R^{X}$, such that $H \subset H^{\prime}$, then $P_{H}=P_{H} P_{H^{\prime}}$. 
Proof. Define $G_{H}: R^{X} \rightarrow H^{*}$ by $\left(G_{H} f\right)(g)=\sum_{x \in X} e^{f(x)} g(x) \mu(x)$ and $\operatorname{grad} F_{H}: H \rightarrow H^{*}$ as in the proof of Theorem 1. We know that $\operatorname{grad} F_{H} \circ$ $G_{H}=G_{H}$ and we have to prove

$$
\operatorname{grad} F_{H} \circ P_{H} \circ P_{H^{\prime}}=G_{H} \text {, or } G_{H} \circ P_{H^{\prime}}=G_{H},
$$

which is trivially true when $H \subset H^{\prime}$.

From now, $H$ is fixed and we denote $\sum_{x \in X} f(x) \mu(x)$ by $\int f d \mu$. Notations are those of Theorem 1.

THEOREM 3. If $H$ contains constant functions of $X$, then:

$$
\int e^{f} f d \mu \geqq \int e^{h_{f}} h_{f} d \mu
$$

and the equality holds only if $f$ is in $H$.

Proof. From the inequality $t \geqq 1-e^{-t}$, for $t$ in $R$ (strict if $t \neq 0$ ), we get

$$
\int e^{f}\left(1-e^{h_{f}-f}\right) d \mu \leqq \int e^{f}\left(f-h_{f}\right) d \mu .
$$

Since $H$ contains constants, the first member of the inequality is 0 (let $g=1$ in the statement of Theorem 1). Now $\int e^{f} h_{f} d \mu=\int e^{h_{s}} h_{f} d \mu$ (let $g=h_{f}$ ), and we are done. Case of equality is clear.

THEOREM 4. Let $h$ in $H$ and $T: X \rightarrow X$. Suppose that either $h \circ T$ is in $H$ or $H$ contains constants and $\mu \circ T=\mu$. Then $h_{h \circ T}=h$ implies $h=h \circ T$.

Proof. If $h \circ T$ is in $H$, we use uniqueness of $h_{f}$ in Theorem 1 . In the other case, we apply Theorem 2 to $f=h \circ T$. But, since $\mu \circ T=\mu$, we have

$$
\int e^{f} f d \mu=\int e^{h} h d \mu
$$

this is the case of equality of Theorem 3.

Remarks. Choose $X=\{1, \cdots, n\} \times\{1, \cdots, m\}, \mu(x) \equiv 1$ and $H$ as in $\S 2$. If $f$ in $\boldsymbol{R}^{X}$ is such that $\int e^{f} d \mu=1$, Theorem 3 gives an inequality about entropy of the joint law of probability, well known in information theory (see [5, pp. 146-157]). If $T$ is a permutation of $X$, of course $\mu \circ T=$ $\mu$; a nice interpretation of the second part of Theorem 4 is as follows.

Let $r=\left(\exp b_{i}\right)_{i=1}^{n}$ and $s=\left(\exp b_{j}^{\prime}\right)_{j=1}^{m}$ such that

$$
\sum_{i=1}^{n} \exp b_{i}=\sum_{j=1}^{n} \exp b_{j}^{\prime}=1 \text {. }
$$


Put the $n m$ numbers $\exp \left(b_{i}+b_{j}^{\prime}\right)$ on slips of paper, try to put them in an $(n, m)$ matrix in order to get an element of $\mathscr{M}(r, s)$; you always get the matrix $\left(\exp \left(b_{i}+b_{j}^{\prime}\right)\right)$, that is to say, the product distribution of probability. Of course we need only Theorem 3, not Theorem 1, to get this result, which can be generalised to a product of countable spaces, with distributions $r$ and $s$ such that $\sum_{i} e^{b_{i}} b_{i}<\infty, \sum_{j} e^{b_{j}^{\prime}} b_{j}^{\prime}<\infty$. I think this is not true if $\sum_{i} e^{b_{i}} b_{i}=\infty$, but I know of no counterexamples.

Considering the exponential in Theorem 1 suffices for applications, but we could replace the exponential by any convex function $\varphi$ positive and increasing, with primitive $\psi$. The proof of Theorem 1 would start from $F(h)=\sum_{x} \psi(h(x)) \mu(x)$.

Generalisations of Theorem 1 to measured spaces $(X, \mu)$ will be done in a forthcoming paper.

\section{BIBLIOGRAPHY}

1. H. Caussinus, Contribution à l'analyse statistique des tableaux de corrélation, Ann. Fac. Sci. Univ. Toulouse (4) 29 (1965), 77-182. MR 39 \#3672.

2. D. J. Hartfiel, Concerning diagonal similarity of irreducible matrices, Proc. Amer. Math. Soc. 30 (1971), 419-425. MR 43 \#7446.

3. D. London, On matrices with a doubly stochastic pattern, J. Math. Anal. Appl. 34 (1971), 648-652. MR 43 \#7448.

4. M. Marcus and M. Newman, The permanent of a symmetric matrix, Notices Amer. Math. Soc. 8 (1961), p. 595.

5. A. Renyi, Foundations of probability, Holden-Day, San Francisco, Calif., 1970. MR 41 \#9314.

6. R. T. Rockafellar, Convex analysis, Princeton Math. Series, no. 28, Princeton Univ. Press, Princeton, N.J., 1970. MR 43 \#445.

7. R. Sinkhorn, A relationship between arbitrary positive matrices and doubly stochastic matrices, Ann. Math. Statist. 35 (1964), 876-879. MR 28 \#5072.

8. - A relationship between arbitrary positive matrices and stochastic matrices, Canad. J. Math. 18 (1966), 303-306. MR 32 \#7574.

9. P. Thionet, Sur certaines variantes des projections du tableau d'échanges interindustriels, Bull. Inst. Internat. Statist. 40 (1963), 431-446.

10. - Note sur le remplissage d'un tableau à double entrée, J. Soc. Statist. Paris, $\mathrm{n}^{\circ} 10,11,12$, (1964), 228-247.

Département de Mathématiques Appliquées, Université de Clermont, B.P. $\mathrm{N}^{\circ} 4563170$ Aubière, France

Current address: U.E.R. de Mathématiques, Université Paul-Sabatier, 118 Route de Narbonne, 31400 Toulouse, France 